\title{
Surgical Outcomes and Clinicopathological Characteristics of Patients Who Underwent Potentially Noncurative Endoscopic Resection for Gastric Cancer: A Report of a Single-Center Experience
}

\author{
Hiroaki Ito, Haruhiro Inoue, Haruo Ikeda, Noriko Odaka, Akira Yoshida, Hitoshi Satodate, \\ Manabu Onimaru, Daisuke Takayanagi, Esperanza Grace Santi, and Shin-ei Kudo
}

Digestive Disease Center, Showa University Northern Yokohama Hospital, 35-1 Chigasakichuo, Tsuzuki-ku, Yokohama 224-8503, Japan

Correspondence should be addressed to Hiroaki Ito; h.ito@med.showa-u.ac.jp

Received 7 March 2013; Accepted 7 May 2013

Academic Editor: Sergio Morini

Copyright (c) 2013 Hiroaki Ito et al. This is an open access article distributed under the Creative Commons Attribution License, which permits unrestricted use, distribution, and reproduction in any medium, provided the original work is properly cited.

Background. Standard treatment of early gastric cancer (EGC) after endoscopic resection with risk factors of nodal metastases and incomplete resection is controversial. We investigated optimal management for the patients with potentially noncurative EGC after endoscopic resection. Methods. We retrospectively examined clinicopathological data and surgical outcomes of all patients with clinically solitary gastric adenocarcinoma who underwent curative surgery after a single peroral endoscopic resection at the Digestive Disease Center of Showa University Northern Yokohama Hospital between April 2001 and December 2012. Fisher's exact test was used for univariate analysis. For multivariate analysis, stepwise multiple linear regression was used to identify independent predictors related to lymph node metastasis and remnant of primary tumor. Results. A total of 41 patients were studied. Four patients (9.8\%) had lymph node metastases. Primary tumors remained in 6 patients (14.6\%). Only venous invasion was statistically significant to lymph node metastasis $(P=0.017)$. With respect to remnant of the primary tumor, pT1b2 tumor invasion $(P=0.015)$ and horizontal margin $(P=0.013)$ were statistically significant. Conclusions. Surgery with limited lymphadenectomy is recommended for tumors with venous invasion or pT1b2 tumor invasion, and additional endoscopic resection may be allowed for tumors with horizontal involvement.

\section{Introduction}

Gastric cancer is the second leading cause of cancer-related death worldwide [1]. The majority of early gastric cancer (EGC) is detected by endoscopic examination. EGC is generally recognized as a tumor with invasion confined to the mucosa or submucosa. The seventh edition of the International Union Against Cancer (UICC) TNM guidelines defines mucosal cancers as Tla and submucosal cancers as pTlb [2]. The third English edition of the Japanese Classification of Gastric Carcinoma (JCG) [3] further categorizes submucosal tumors as pT1b1 (submucosal invasion of $<0.5 \mathrm{~mm}$ ) or pTlb2 (submucosal invasion of $\geq 0.5 \mathrm{~mm}$ ). Because nodal metastases are rare in pTla tumors $[4,5]$, per-oral endoscopic tumor resection, such as endoscopic mucosal resection (EMR) and endoscopic submucosal dissection (ESD), has been widely performed for clinical Tla tumors. In Japan, because undifferentiated EGC has a relatively higher incidence of lymph node metastasis than does differentiated EGC, EMR and ESD have not been recommended for undifferentiated EGC [6] with the exception of tumors of $<20 \mathrm{~mm}$ without ulceration $[7,8]$. However, the depth of invasion of resected tumors is often diagnosed as pTlb or deeper. Nodal metastases occur in $2 \%$ to $9.8 \%$ of pT1b1 and $12 \%$ to $24.3 \%$ of pT1b2 tumors $[9,10]$. Lymphatic and venous invasions are reported as significant risk factors of lymph node metastases [10]. Undifferentiated tumors, including mucinous carcinoma, signet-ring cell carcinoma, and poorly differentiated adenocarcinoma [10], and tumors of $>3 \mathrm{~cm}$ [11] are also reported as risk factors for lymph node metastases. Thus, additional surgical resection 
including lymphadenectomy is recommended for pT1b or deeper tumors, tumors with lymphatic and venous invasions, and tumors mainly composed of undifferentiated carcinoma, although the majority of patients who undergo additional surgery may have no nodal metastasis [10].

On the other hand, additional surgical resection for tumors that were incompletely resected by endoscopic resection is controversial. JCG defines the horizontal margin (HM) and vertical margin (VM) as HM1 and VM1 (margin involvement), HM0 and VM0 (no margin involvement) or HMX and VMX (involvement of the margin cannot be assessed) [3]. Although additional endoscopic resection is recommended for HM1 tumors [12, 13] and surgical resection is recommended for VM1 tumors [12,13], there is a possibility that no primary tumor will be present in the surgical specimen.

The aim of this study was to investigate the optimal treatment strategy for gastric cancer after endoscopic resection with risk factors of nodal metastases and incomplete resection. We estimated the relationship between clinicopathological findings of specimens from endoscopic and surgical resection and evaluated risk factors for lymph node metastases and remnant of the primary tumor.

\section{Patients and Methods}

2.1. Patients. All patients with gastric adenocarcinoma who underwent curative surgery after single per-oral endoscopic resection at the Digestive Disease Center of Showa University Northern Yokohama Hospital between April 2001 and December 2012 were retrospectively studied. The criteria for inclusion in the study were (1) a clinically solitary tumor, (2) histologically proven adenocarcinoma, (3) no prior treatment before endoscopic resection, and (4) noncurative endoscopic resection defined by the Japanese gastric cancer treatment guidelines 2010 (version 3) [14]. Patients with synchronous or metachronous malignancy were excluded.

The formalin-fixed specimens resected by endoscopic resection were cut into multiple slices in parallel with the lesser curvature at a $5 \mathrm{~mm}$ interval or adequate width depending on tumor or mucosal scar size. Hematoxylin and eosin and immunohistochemical staining using antibodies of D240 (Dako-Japan, Tokyo, Japan) and Victoria Blue (DakoJapan, Tokyo, Japan) were performed to detect lymphatic and venous invasions. The formalin-fixed lymph nodes were cut into two pieces, and the cut surfaces were examined. The histological diagnosis was determined by two or more examiners, including at least one board-certified pathologist, following the UICC classification and JCG. We reviewed the clinical records and histological reports to examine relationships between the histological diagnosis of specimens from endoscopic and surgical resection and the prognosis.

2.2. Statistical Analysis. Fisher's exact test was used for univariate analysis. Variables showing a univariate association $(P<0.50)$ were also subjected to multivariate analysis. For multivariate analysis, stepwise multiple linear regression was used to identify independent predictors related to lymph node metastasis and remnant of primary tumor. $P$ values of $<0.05$ were considered to indicate statistical significance. Statistical analysis was performed using JMP 9.0.2 (SAS Institute, Cary, NC, USA).

The study was approved by the Institutional Review Board of Showa University, Northern Yokohama Hospital (number 1203-03). The research reported in this paper was in compliance with the Helsinki Declaration. This study was registered with the University Hospital Medical Information Network in Japan (number UMIN000007430).

\section{Results}

A total of 41 patients were eligible for inclusion in the study, including 38 males and 3 females with a mean age of 67.7 years (range, 46-83 years). The median interval between endoscopic resection and surgery was 2 months (range, 0 5 months). The median follow-up period after surgery was 23 months. No patient experienced recurrent disease after surgery.

The clinicopathological characteristics of patients are shown in Table 1. Nine and 32 of 41 patients underwent endoscopic mucosal resection and endoscopic submucosal dissection, respectively. Reasons for surgery were risk of lymph node metastasis (submucosal invasion, venous and lymphatic invasions, and undifferentiated component) and incomplete tumor resection (horizontal and vertical margin involvement). Among the 41 patients, partial, proximal, distal, and total gastrectomies were performed in 5, 9, 20, and 7 patients, respectively.

Four (9.8\%) of 41 patients had lymph node metastases in perigastric nodes. Primary tumors remained in $6(14.6 \%)$ of the 41 patients, none of whom had metastatic nodes. Relationships among clinicopathological characteristics, nodal metastasis, and remnant of the primary tumor are summarized in Table 2.

Four of 37 tumors with submucosal invasion (4 of 33 pT1b2 tumors), 3 of 20 with lymphatic invasion, and 4 of 18 with venous invasion had metastatic nodes. Five of 37 tumors with submucosal invasion ( 3 of 33 pTlb2 tumors), 4 of 20 with lymphatic invasion, 1 of 18 with venous invasion, 3 of 4 categorized as HM1, and 4 of 14 categorized as VM1 had remnants of primary tumors.

In univariate analysis, venous invasion was the only significant factor for nodal metastasis $(P=0.047)$. Tumor size $(P=0.445)$, depth of tumor invasion $(P=0.404)$, ulceration $(P=0.582)$, main histologic type $(P=0.517)$, and lymphatic invasion $(P=0.284)$ were not significant factors for nodal metastasis.

For remnant of the primary tumor, endoscopic procedure $(P=0.015)$ and horizontal margin $(P=0.001)$ were significant factors. Depth of tumor invasion $(P=0.077)$, venous invasion $(P=0.157)$, and vertical margin $(P=0.228)$ had weak relationships.

The results of the multivariate analysis are summarized in Table 3. Multivariate analysis showed that only venous invasion was significant with lymph node metastasis $(P=$ $0.017)$. Age $(P=0.545)$, endoscopic treatment $(P=0.264)$, pathological tumor size $(P=0.482)$, depth of tumor invasion 
TABLE 1: Clinicopathological findings of patients $(n=41)$.

\begin{tabular}{|c|c|}
\hline Variables & Number of subjects (\%) \\
\hline \multicolumn{2}{|l|}{ Sex } \\
\hline Male & $38(92.7)$ \\
\hline Female & $3(7.3)$ \\
\hline \multicolumn{2}{|l|}{ Endoscopic treatment } \\
\hline Mucosal resection & $9(22.0)$ \\
\hline Submucosal dissection & $32(78.0)$ \\
\hline \multicolumn{2}{|l|}{ Surgical indication } \\
\hline Submucosal tumor invasion & $37(90.2)$ \\
\hline Lymphatic or venous invasion & $27(65.9)$ \\
\hline Undifferentiated component $^{\dagger}$ & $24(58.5)$ \\
\hline Horizontal margin involvement & $4(9.8)$ \\
\hline Vertical margin involvement & $14(34.1)$ \\
\hline \multicolumn{2}{|l|}{ Surgical approach } \\
\hline Laparoscopy & $34(82.9)$ \\
\hline Hand assistance & $4(9.8)$ \\
\hline Open laparotomy & $3(7.3)$ \\
\hline \multicolumn{2}{|l|}{ Extent of gastrectomy } \\
\hline Partial & $5(12.2)$ \\
\hline Proximal & $9(22.0)$ \\
\hline Distal & $20(48.8)$ \\
\hline Total & $7(17.1)$ \\
\hline \multicolumn{2}{|l|}{$\begin{array}{l}\text { Remnant of primary tumor (surgical } \\
\text { specimen) }\end{array}$} \\
\hline No & $35(85.4)$ \\
\hline Yes & $6(14.6)$ \\
\hline \multicolumn{2}{|l|}{ Depth of tumor invasion ${ }^{\ddagger}$} \\
\hline pTla & $4(9.8)$ \\
\hline pT1b1 & $4(9.8)$ \\
\hline pT1b2 & $33(80.5)$ \\
\hline \multicolumn{2}{|l|}{$\begin{array}{l}\text { Lymph node metastasis (surgical } \\
\text { specimen) }\end{array}$} \\
\hline pNo & $37(90.2)$ \\
\hline $\mathrm{pN} 1$ & $4(9.8)$ \\
\hline \multicolumn{2}{|l|}{ TNM stage (surgical specimen) } \\
\hline fStage IA & $37(90.2)$ \\
\hline fStage IB & $4(9.8)$ \\
\hline
\end{tabular}

${ }^{\dagger}$ Differentiated: well-differentiated adenocarcinoma, moderately differentiated adenocarcinoma, or papillary adenocarcinoma; undifferentiated: poorly differentiated adenocarcinoma, signet-ring cell adenocarcinoma, or mucinous adenocarcinoma.

${ }^{\ddagger}$ According to the third English edition of the Japanese Classification of Gastric Carcinoma.

( $P=0.300)$, macro type $(P=0.121)$, lymphatic invasion $(P=$ $0.269)$, and vertical margin $(P=0.482)$ were not significant. For remnant of the primary tumor, depth of tumor invasion (pT1b2 tumor invasion) $(P=0.015)$ and horizontal margin $(P=0.013)$ were significant. Sex $(P=0.365)$, endoscopic treatment $(P=0.071)$, pathological tumor size $(P=0.836)$, lymphatic invasion $(P=0.159)$, venous invasion $(P=0.091)$, and vertical margin $(P=0.072)$ were not significant.

\section{Discussion}

The most effective treatment for gastric cancer is surgery, including gastrectomy and lymphadenectomy [15]. However, surgery is often overtreatment, especially for EGC without nodal metastasis. If we estimate that the risk of nodal metastasis is very small, endoscopic treatment without lymphadenectomy (e.g., EMR and ESD) can be performed for EGC [16-18]. Depth of tumor invasion, ulceration, lymphatic and venous invasion, and undifferentiated component have been reported as risk factors of lymph node metastasis $[4,10]$. When these risk factors are detected by pathological examination, surgical treatment with lymphadenectomy is generally added [11], despite the fact that the incidence of nodal metastases of EGC is rare [3]. EGC is a curable disease, but inadequate treatment may yield remnant metastatic lymph nodes. The Japanese Gastric Cancer Association created a criterion for safe endoscopic treatment [14]. As a result, some patients without nodal metastasis may undergo unnecessary surgical operations.

In this study, the incidence of nodal metastases in tumors with non-curative potential after endoscopic resection was $9.8 \%$. Statistically, only venous invasion was independently significant with nodal metastases in terms of pathological characteristics, and there was no nodal metastasis in the tumors without pathological venous invasion. Moreover, only 1 of 21 tumors without lymphatic invasion had nodal metastasis. In terms of the other pathological factors, mucosal tumors had no nodal metastasis, and ulceration and histological type had no significant relationship with nodal metastasis. Differences in risk factors for nodal metastasis exist between previous reports and the present study. These discrepancies were caused by differences in specimens. The risk factors of nodal metastases have been based on pathological findings of surgical specimens. In this study, we investigated specimens obtained by endoscopic resection. These findings should be confirmed in a large-scale study.

On the other hand, positive tumor involvement (HM1 and VM1) in endoscopically resected specimens is also generally considered to be an indication for additional endoscopic resection [13] or surgery $[12,13,19]$. However, the absence of pathological carcinoma in the surgical specimens was not rare. Oda et al. reported that $47.4 \%$ of surgical specimens containing tumors with a possible lateral margin had residual tumors [12]. It is thought that achieving an exact diagnosis with endoscopically resected specimens is sometimes difficult [20] because these specimens are histologically modified.

In our study, 3 of $4(75.0 \%)$ patients with an HM1 tumor, 4 of $14(28.6 \%)$ with a VM1 tumor, and 1 of $25(4.0 \%)$ without evidence of margin involvement had a remnant of the primary tumor. In the statistical analysis, horizontal margin $(P=0.013)$ and pT1b2 tumor invasion $(P=0.015)$ were independently significant factors of remnants primary tumors. Vertical margin was not significant $(P=0.072)$. The endoscopic treatment, whether EMR or ESD, was also not significant. Because all 12 patients with remnants of 
TABLE 2: Results of univariate analyses showing relationships among clinicopathological characteristics of specimens.

\begin{tabular}{|c|c|c|c|c|}
\hline \multirow{2}{*}{ Variables } & \multicolumn{2}{|c|}{ Lymph node metastasis } & \multicolumn{2}{|c|}{ Remnant of primary tumor } \\
\hline & Subject & $P$ value & Subject & $P$ value \\
\hline Total & $4 / 41(9.8 \%)$ & & $6 / 41(14.6 \%)$ & \\
\hline Sex & & 0.729 & & 0.386 \\
\hline Male & $4 / 38(10.5 \%)$ & & $5 / 38(13.2 \%)$ & \\
\hline Female & $0 / 3$ & & $1 / 3(33.3 \%)$ & \\
\hline Age (years) & & 0.488 & & 0.566 \\
\hline$\leq 70$ & $3 / 25(12.0 \%)$ & & $4 / 25(16.0 \%)$ & \\
\hline$>70$ & $1 / 16(6.3 \%)$ & & $2 / 16(12.5 \%)$ & \\
\hline Endoscopic treatment & & 0.355 & & $0.015^{*}$ \\
\hline Mucosal resection & $0 / 9$ & & $4 / 9(44.4 \%)$ & \\
\hline Submucosal dissection & $4 / 32(12.5 \%)$ & & $2 / 32(6.3 \%)$ & \\
\hline Tumor size (mm) & & 0.445 & & 0.488 \\
\hline$\leq 20$ & $3 / 24(12.5 \%)$ & & $3 / 24(12.5 \%)$ & \\
\hline$>20$ & $1 / 17(5.9 \%)$ & & $3 / 17(17.6 \%)$ & \\
\hline Depth of tumor invasion ${ }^{\dagger}$ & & 0.404 & & 0.077 \\
\hline pTla-1b1 & $0 / 8$ & & $3 / 8(37.5 \%)$ & \\
\hline pT1b2 & $4 / 33(12.1 \%)$ & & $3 / 33(9.1 \%)$ & \\
\hline Macrotype & & 0.156 & & 0.566 \\
\hline Elevated & $3 / 16(18.8 \%)$ & & $2 / 16(12.5 \%)$ & \\
\hline Depressed & $1 / 25(4.0 \%)$ & & $4 / 25(16.0 \%)$ & \\
\hline Intratumoral ulceration or ulcer scar & & 0.582 & & 0.852 \\
\hline No & $4 / 36(11.1 \%)$ & & $5 / 36(13.9 \%)$ & \\
\hline Yes & $0 / 5$ & & $1 / 5(20.0 \%)$ & \\
\hline Main histologic type $\mathrm{e}^{\ddagger}$ & & 0.517 & & 0.639 \\
\hline Differentiated & $4 / 35(11.4 \%)$ & & $5 / 35(14.3 \%)$ & \\
\hline Undifferentiated & $0 / 6$ & & 1/6 (16.7\%) & \\
\hline Undifferentiated component ${ }^{\ddagger}$ & & 0.578 & & 0.672 \\
\hline No & $3 / 27(11.1 \%)$ & & $4 / 27(14.8 \%)$ & \\
\hline Yes & $1 / 14(7.1 \%)$ & & $2 / 14(14.3 \%)$ & \\
\hline Lymphatic invasion & & 0.284 & & 0.307 \\
\hline L0 & $1 / 21(4.8 \%)$ & & $2 / 21(9.5 \%)$ & \\
\hline $\mathrm{L} 1$ & $3 / 20(15.0 \%)$ & & $4 / 20(20.0 \%)$ & \\
\hline Venous invasion & & $0.030^{*}$ & & 0.157 \\
\hline V0 & $0 / 23$ & & $5 / 23(21.7 \%)$ & \\
\hline V1 & $4 / 18(22.2 \%)$ & & $1 / 18(5.6 \%)$ & \\
\hline Horizontal margin $^{\dagger}$ & & 0.582 & & $0.001^{* *}$ \\
\hline HM0 or HMX & $4 / 37(10.8 \%)$ & & $3 / 37(8.1 \%)$ & \\
\hline HM1 & $0 / 4$ & & $3 / 4(75.0 \%)$ & \\
\hline Vertical margin ${ }^{\dagger}$ & & $0.047^{*}$ & & 0.228 \\
\hline VM0 or VMX & $2 / 27(7.4 \%)$ & & $2 / 27(7.4 \%)$ & \\
\hline VM1 & $2 / 14(14.3 \%)$ & & $4 / 14(28.6 \%)$ & \\
\hline
\end{tabular}

${ }^{*} P<0.05,{ }^{* *} P<0.01$.

${ }^{\dagger}$ According to the third English edition of the Japanese Classification of Gastric Carcinoma.

${ }^{\ddagger}$ Differentiated: well-differentiated adenocarcinoma, moderately differentiated adenocarcinoma, or papillary adenocarcinoma; undifferentiated: poorly differentiated adenocarcinoma, signet-ring cell adenocarcinoma, or mucinous adenocarcinoma.

primary tumors had no nodal metastases, it was suggested that tumor involvement is not always a necessary condition for additional lymphadenectomy. Thus, additional resection may be performed endoscopically.
In our opinion, when non-curative endoscopic resection is considered, additional surgical resection including lymphadenectomy should be performed for tumors with venous invasion. If tumors with horizontal involvement 
TABLE 3: Results of multivariate analyses showing relationships among clinicopathological characteristics of the specimens.

\begin{tabular}{lclc}
\hline \multicolumn{1}{c}{ Lymph node metastasis } & & \multicolumn{2}{c}{ Remnant of primary tumor } \\
Variables & $P$ value & Variables & $P$ value \\
\hline Age & 0.545 & Sex & 0.365 \\
Endoscopic treatment & 0.264 & Endoscopic treatment & 0.071 \\
Pathological tumor size & 0.482 & Pathological tumor size & 0.836 \\
Depth of tumor invasion & 0.300 & Depth of tumor invasion & $0.015^{*}$ \\
Macrotype & 0.121 & Lymphatic invasion & 0.159 \\
Lymphatic invasion & 0.269 & Venous invasion & 0.091 \\
Venous invasion & $0.017^{*}$ & Horizontal margin & $0.013^{*}$ \\
Vertical margin & 0.482 & Vertical margin & 0.072 \\
${ }^{*} P<0.05$ & & &
\end{tabular}

have no venous invasion, endoscopic resection without lymphadenectomy may be allowed. Because $10 \%$ of submucosal tumors have nodal metastases, surgical resection including lymphadenectomy should be performed for tumors with vertical involvement and pT1b2 (submucosal invasion of $\geq 0.5 \mathrm{~mm}$ ) tumors.

\section{Conclusions}

We studied the clinicopathological data and surgical outcomes of 41 patients with potentially non-curative EGC after endoscopic resection. Reasons for surgery were risk of lymph node metastasis and incomplete tumor resection. Four and 6 tumors had lymph node metastases and remnants of primary tumors after additional curative surgery, respectively. In terms of the pathological characteristics of the endoscopically resected specimens, venous invasion was the only significant risk factor for nodal metastasis $(P=0.017)$. Tumors without venous invasion had no nodal metastasis. On the other hand, pT1b2 (submucosal invasion of $\geq 0.5 \mathrm{~mm}$ ) tumor invasion $(P=0.015)$ and horizontal margin $(P=0.013)$ were significantly related to remnants of primary tumors. Sex, age, endoscopic treatment, pathological tumor size, histological type, ulceration, lymphatic invasion, and vertical margin had no significant relationship with either nodal metastasis or remnants of primary tumors. According to our data, surgery with limited lymphadenectomy is recommended for tumors with venous invasion or vertical involvement and pT1b2 tumors, and additional endoscopic resection may be allowed for tumors with horizontal involvement.

\section{Abbreviations}

EGC: Early gastric cancer

UICC: International Union Against Cancer

JCG: Japanese Classification of Gastric Carcinoma

EMR: Endoscopic mucosal resection

ESD: Endoscopic submucosal dissection

HM: Horizontal margin

VM: Vertical margin.

\section{Conflict of Interests}

The authors declare that they have no conflict of interests.

\section{Authors' Contribution}

H. Ito conceived and designed the study, collected clinical and pathological data, performed the statistical analysis, and interpreted the data. $H$. Inoue performed medical examinations, collected biopsy samples, performed the surgical operations, and interpreted the data. H. Ikeda, A. Yoshida, M. Onimaru, and E. G. Santi performed medical examinations and collected samples. N. Odaka, H. Satodate, and D. Takayanagi performed medical examinations and surgical operations. S. Kudo participated in the study design and coordination. All authors have read and approved the final paper.

\section{Acknowledgments}

The authors are extremely grateful to all of the patients. They are also most grateful to the clinical staff.

\section{References}

[1] D. M. Parkin, F. Bray, J. Ferlay, and P. Pisani, "Global cancer statistics, 2002," CA: A Cancer Journal for Clinicians, vol. 55, no. 2, pp. 74-108, 2005.

[2] L. H. Sobin, M. K. Gospodarowicz, and C. Wittekind, TNM Classification of Malignant Tumors, Wiley-Blackwell, Oxford, UK, 7th edition, 2010.

[3] Japanese Gastric Cancer Association, "Japanese classification of gastric carcinoma: 3rd English edition," Gastric Cancer, vol. 14, no. 2, pp. 101-112, 2011.

[4] T. Gotoda, A. Yanagisawa, M. Sasako et al., "Incidence of lymph node metastasis from early gastric cancer: estimation with a large number of cases at two large centers," Gastric Cancer, vol. 3, no. 4, pp. 219-225, 2000.

[5] T. Yamao, K. Shirao, H. Ono et al., "Risk factors for lymph node metastasis from intramucosal gastric carcinoma," Cancer, vol. 77, no. 4, pp. 602-606, 1996.

[6] T. Gotoda, H. Yamamoto, and R. M. Soetikno, "Endoscopic submucosal dissection of early gastric cancer," Journal of Gastroenterology, vol. 41, no. 10, pp. 929-942, 2006.

[7] N. Abe, T. Watanabe, M. Sugiyama et al., "Endoscopic treatment or surgery for undifferentiated early gastric cancer?" The American Journal of Surgery, vol. 188, no. 2, pp. 181-184, 2004.

[8] T. Hirasawa, T. Gotoda, S. Miyata et al., "Incidence of lymph node metastasis and the feasibility of endoscopic resection for 
undifferentiated-type early gastric cancer," Gastric Cancer, vol. 12, no. 3, pp. 148-152, 2009.

[9] N. Kurihara, T. Kubota, Y. Otani et al., "Lymph node metastasis of early gastric cancer with submucosal invasion," The British Journal of Surgery, vol. 85, no. 6, pp. 835-839, 1998.

[10] T. Gotoda, M. Sasako, H. Ono, H. Katai, T. Sano, and T. Shimoda, "Evaluation of the necessity for gastrectomy with lymph node dissection for patients with submucosal invasive gastric cancer," The British Journal of Surgery, vol. 88, no. 3, pp. 444449, 2001.

[11] K. W. Ryu, I. J. Choi, Y. W. Doh et al., "Surgical indication for non-curative endoscopic resection in early gastric cancer," Annals of Surgical Oncology, vol. 14, no. 12, pp. 3428-3434, 2007.

[12] I. Oda, T. Gotoda, M. Sasako et al., "Treatment strategy after non-curative endoscopic resection of early gastric cancer," The British Journal of Surgery, vol. 95, no. 12, pp. 1495-1500, 2008.

[13] J. H. Lee, J. H. Kim, D. H. Kim, T. Y. Jeon, G. H. Kim, and Y. Park do, "Is surgical treatment necessary after non-curative endoscopic resection for early gastric cancer?" Journal of Gastric Cancer, vol. 10, no. 4, pp. 182-187, 2010.

[14] Japanese Gastric Cancer Association, "Japanese gastric cancer treatment guidelines 2010 (ver. 3)," Gastric Cancer, vol. 14, no. 2, pp. 113-123, 2011.

[15] M. Degiuli, M. Sasako, A. Ponti, T. Soldati, F. Danese, and F. Calvo, "Morbidity and mortality after D2 gastrectomy for gastric cancer: Results of the Italian gastric cancer study group prospective multicenter surgical study," Journal of Clinical Oncology, vol. 16, no. 4, pp. 1490-1493, 1998.

[16] K. Takeshita, M. Tani, H. Inoue et al., "Endoscopic treatment of early oesophageal or gastric cancer," Gut, vol. 40, no. 1, pp. 123-127, 1997.

[17] H. Ono, H. Kondo, T. Gotoda et al., "Endoscopic mucosal resection for treatment of early gastric cancer," Gut, vol. 48, no. 2, pp. 225-229, 2001.

[18] H. Ono, "Endoscopic submucosal dissection for early gastric cancer," Chinese Journal of Digestive Diseases, vol. 6, no. 3, pp. 119-121, 2005.

[19] H. Jung, J. M. Bae, M. G. Choi, J. H. Noh, T. S. Sohn, and S. Kim, "Surgical outcome after incomplete endoscopic submucosal dissection of gastric cancer," The British Journal of Surgery, vol. 98, no. 1, pp. 73-78, 2011.

[20] T. Gotoda, "A large endoscopic resection by endoscopic submucosal dissection procedure for early gastric cancer," Clinical Gastroenterology and Hepatology, vol. 3, supplement 1, pp. S71S73, 2005. 


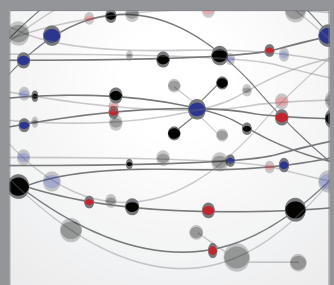

The Scientific World Journal
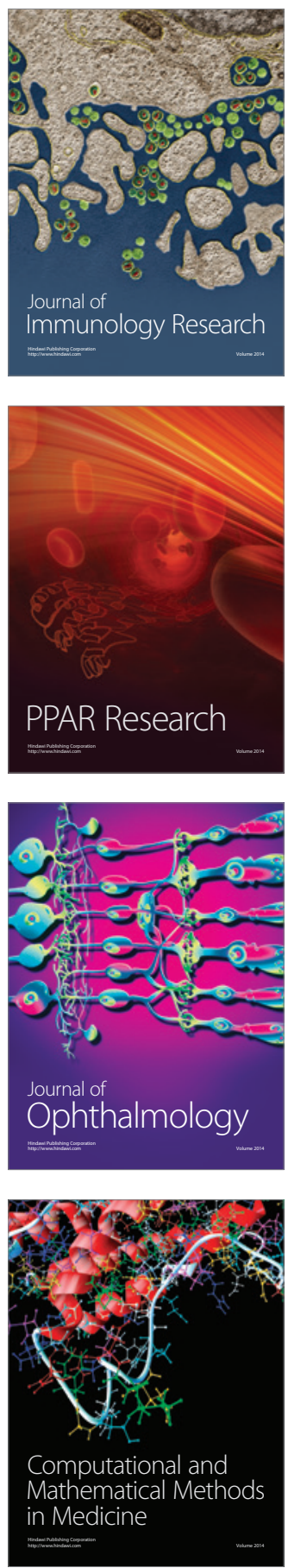

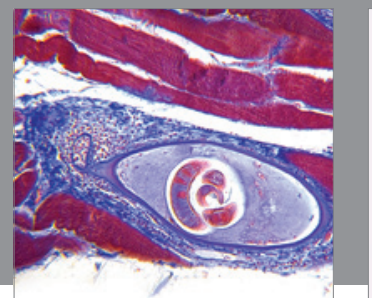

Gastroenterology

Research and Practice
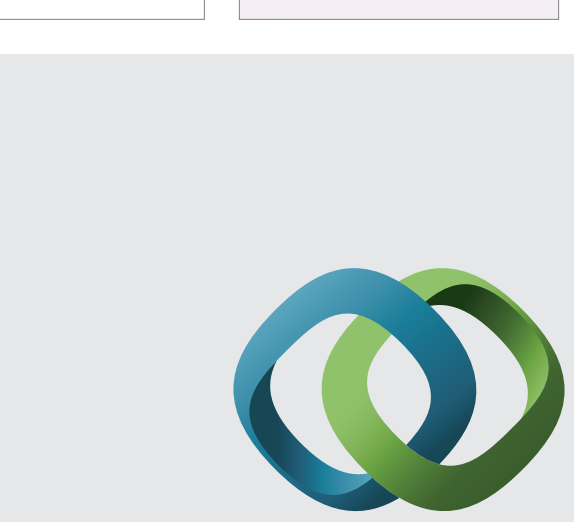

\section{Hindawi}

Submit your manuscripts at

http://www.hindawi.com
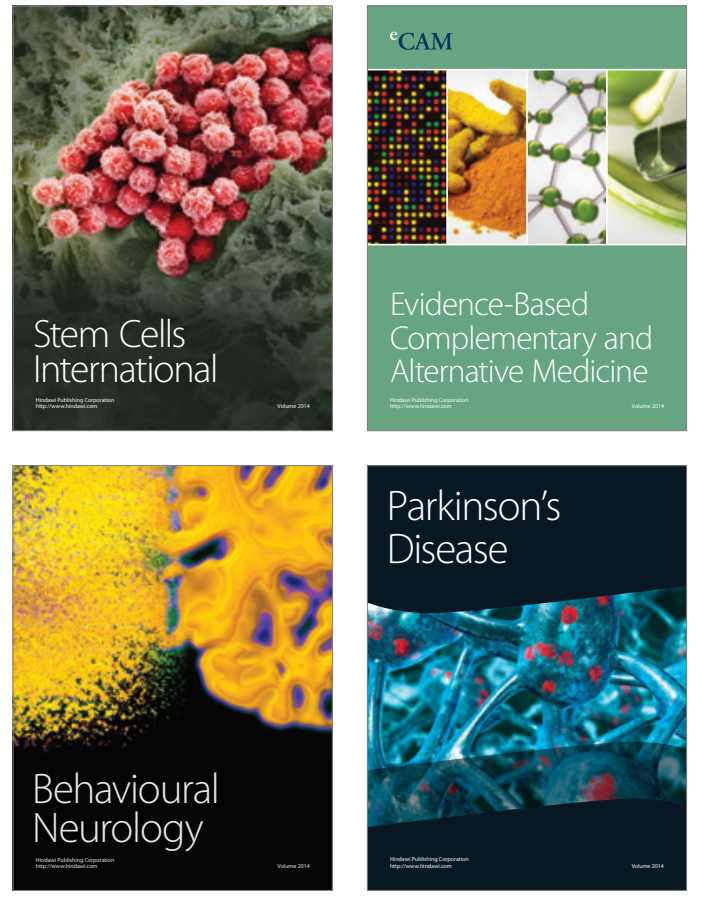
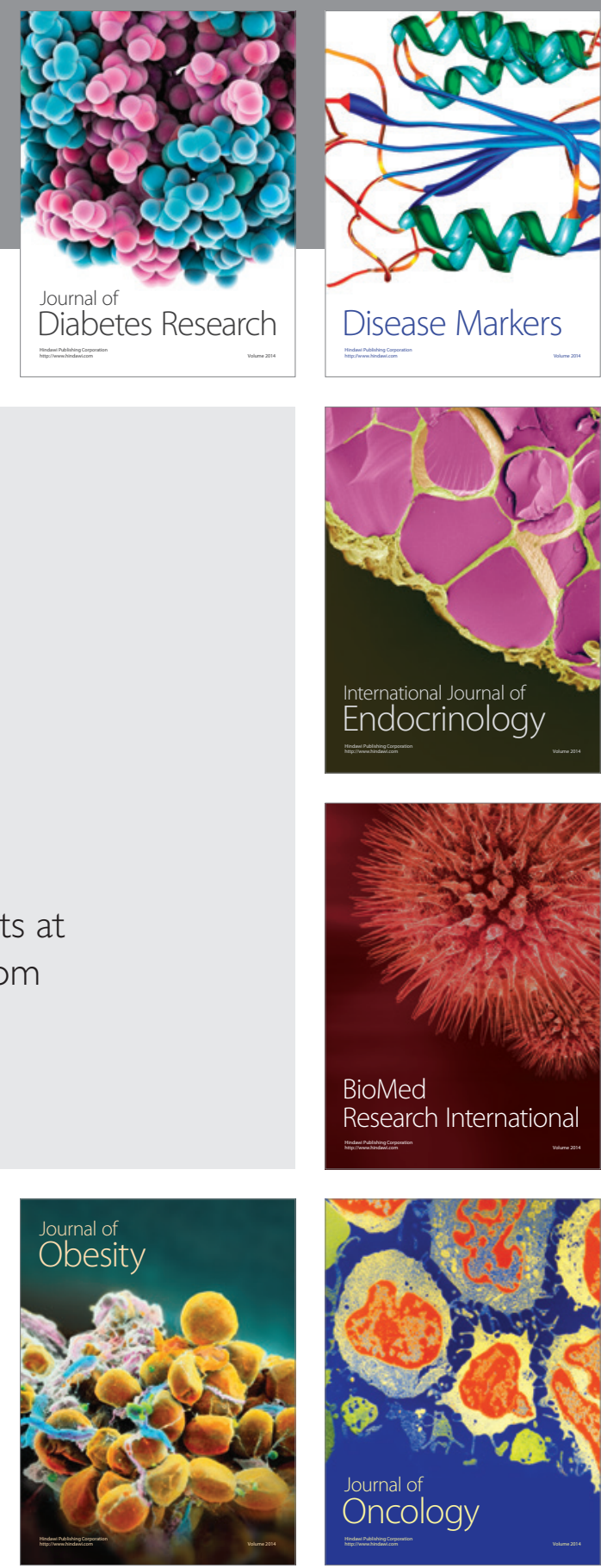

Disease Markers
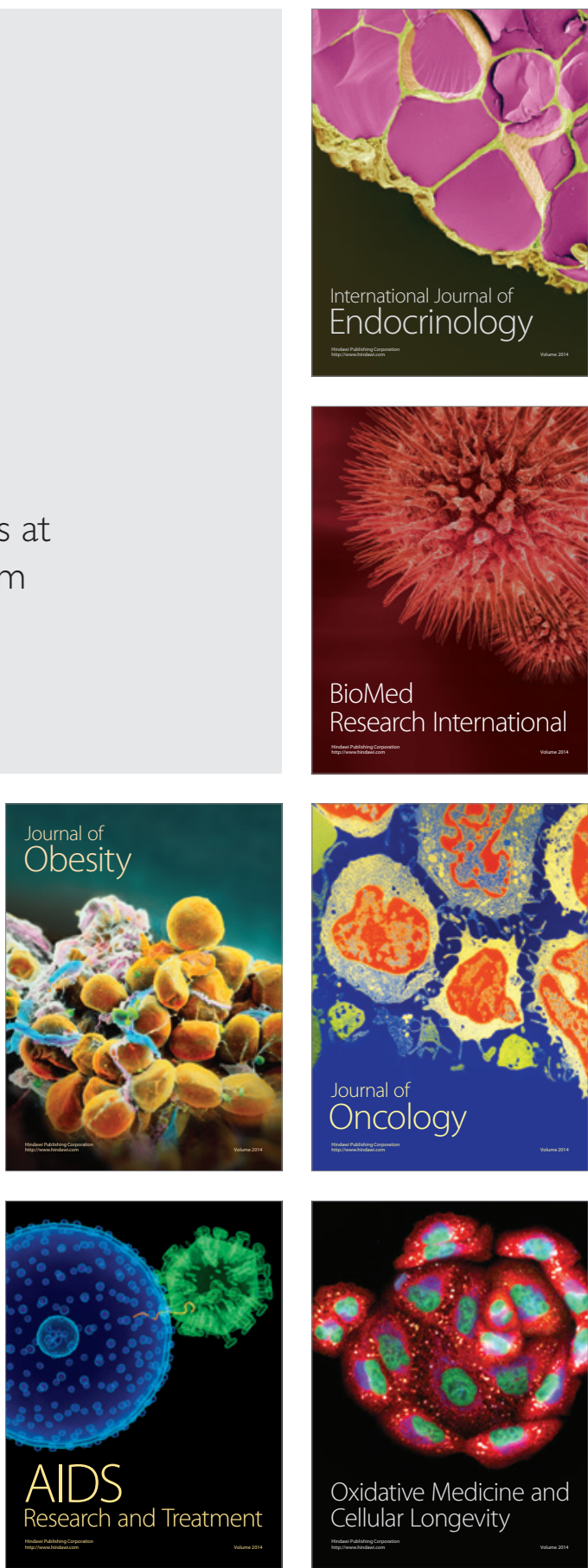